\title{
Overexpression of DJ-I correlates with aggressive clinicopathological characteristics and poor prognosis in malignant tumors: a meta-analysis
}

This article was published in the following Dove Press journal:

OncoTargets and Therapy

\author{
Qingting Wang \\ Fangwei Li \\ Wenhua Shi \\ Qianqian Zhang \\ Jian Wang \\ Xin Yan \\ Limin Chai \\ Manxiang $\mathrm{Li}$
}

Department of Respiratory and Critical Care Medicine, The First Affiliated Hospital of Xi'an Jiaotong University, Xi'an, Shaanxi 71006I, China
Correspondence: Manxiang $\mathrm{Li}$ Department of Respiratory and Critical Care Medicine, The First Affiliated Hospital of Xi'an Jiaotong University, No 277, West Yanta Road, Xi'an, Shaanxi 71006I, China

Tel/fax +860298532 4053

Email manxiangli@hotmail.com
Purpose: A number of studies have investigated the role of DJ-1 in the development and progression of malignant tumors. This meta-analysis aims to systematically estimate the relationship between the expression level of DJ-1 and the malignant biological behaviors of tumors and to assess the clinical significances of DJ-1 in the prognosis and diagnosis of cancer.

Materials and methods: We searched PubMed, Web of Science, China National Knowledge Infrastructure and Wanfang databases from inception to December 1, 2017. Pooled odds ratio (OR) and hazard ratio (HR) with their 95\% confidence interval and the diagnostic value of DJ-1 were calculated.

Results: Fourteen eligible studies with a total of 1,947 subjects were enrolled in our metaanalysis. The results showed that DJ-1 was overexpressed in cancer patients compared with noncancer patients $(\mathrm{OR}=30.72)$, and elevated expression of DJ-1 was demonstrated to be closely associated with high tumor-node-metastasis stage $(\mathrm{OR}=5.52)$, poor differentiated degree $(\mathrm{OR}=2.46)$, positive lymph node metastasis $(\mathrm{OR}=4.12)$ and worse overall survival $(H R=2.23)$. In addition, the combined sensitivity and specificity for DJ-1 to discern malignant tumors were 0.73 and 0.93 , respectively. The diagnostic OR was 34.87 , and the area under the summary receiver operating characteristic curve was 0.88 .

Conclusion: This meta-analysis demonstrated that DJ-1 was an important biomarker in tumor assessment and prognosis prediction.

Keywords: DJ-1, prognosis, diagnosis, malignant tumor, meta-analysis

\section{Introduction}

Cancer displays an increasing incidence and mortality rate and emerges as a major public health problem worldwide. According to the Cancer Statistics, 1,688,780 new cancer cases and 600,920 cancer deaths are predicted to happen in the USA in $2017 .{ }^{1}$ Although remarkable advances have been made in several diagnostic methods and therapeutic techniques, the overall survival among cancer patients remains poor. ${ }^{2}$ Since tumor biomarkers show great potential to identify cancer risk, promote early detection and provide therapeutic target, its development has been considered as an important part to improve patient outcomes in oncology. ${ }^{3}$ However, despite a great number of tumor biomarkers have been discovered and validated in recent years, the limitations in early diagnosis and the lack of effectiveness of these biomarkers result in that only a handful of biomarkers can be approved for clinical practices. ${ }^{4}$ Therefore, it is an urgent need to explore sensitive biomarkers that have good performances in predicating clinicopathological features and discerning malignant tumors. 
DJ-1 (PARK7), a novel mitogen-dependent oncogene, locates on the distal part of the short arm of chromosome 1 (1p36.12-1p36.33), where it has many chromosome aberrations in cancers. ${ }^{5}$ It has been demonstrated that DJ-1 participates in many aspects of biological processes, including carcinogenesis and tumor progression. ${ }^{6}$ Study by Kim et al has found that DJ-1 causes tumor cell proliferation and invasion by inhibiting the effect of the tumor suppressor gene phosphatase and tensin homolog (PTEN) through PI3K survival pathway. ${ }^{7}$ In addition, DJ-1 is a multifunctional antioxidant protein that senses reactive oxygen species levels and determines the cell fate by regulating autophagy and apoptosis in cancers. ${ }^{8}$ Downregulation of DJ-1 suppresses cell growth and improves the sensitivity to chemotherapies. ${ }^{9}$ Moreover, an increasing number of studies have indicated that DJ-1 protein is overexpressed in various types of cancer, such as glottis squamous cell carcinoma (GSCC), ${ }^{10}$ nonsmall-cell lung carcinoma, ${ }^{11}$ breast cancer (BC), ${ }^{12-15}$ gastric carcinoma (GC), ${ }^{16}$ hepatocellular carcinoma (HCC), ${ }^{17}$ pancreatic ductal adenocarcinoma ${ }^{18}$ and prostate cancer. ${ }^{19}$ Besides, high expression of DJ-1 is significantly associated with poor prognosis in malignant tumors. ${ }^{10,17,20-23}$ Given that a single study may lack the power to provide reliable conclusions because of the small sample size and methodological limitations, we performed a systematic and comprehensive meta-analysis to clarify the significance of DJ-1 in describing the clinicopathological characteristics of malignant tumors and to assess the value of DJ-1 in cancer prognosis and diagnosis, thereby to further explore whether DJ-1 could be used as a reliable molecular marker in clinical uses.

\section{Materials and methods Search strategy}

This meta-analysis followed the standard guidelines of Preferred Reporting Items for Systematic Reviews and Meta-Analysis. ${ }^{24}$ We systematically searched the published literatures from the electronic databases of PubMed, Web of Science, Chinese National Knowledge Infrastructure and Wanfang database up to December 1, 2017, and used ("DJ-1" or "PARK7" or "DJ-1-PARK7 Protein" or "DJ-1 Protein" or "Parkinson Protein 7" or "Parkinsonism Associated Deglycase") AND ("Neoplasia" or "Neoplasm" or "Tumors" or "Cancer" or "Carcinoma" or "Malignant neoplasms" or "Malignant tumors") as free text and Medical Subject Heading terms to improve the searching sensitivity. Manual search of citations from relevant original studies and review articles was also performed.
The languages of publications were limited to English and Chinese. Any disagreements were seriously discussed and finally solved by consensus.

\section{Selection criteria}

The inclusion criteria of this meta-analysis were 1) the data about DJ-1 expression level in tumor tissue was abundant; 2) case-control or cohort studies; 3 ) the sample size not less than 50;4) pathologically confirmed patients and 5) the tumor tissues were taken before any treatments. Studies were excluded according to the following criteria: 1) articles not supply original data such as reviews, editorials, letters, case reports and expert opinions; 2) not supply sufficient or useful data about DJ-1 expression level; 3) nonhuman studies and 4) duplicated or repeated studies.

\section{Data extraction}

The following information were collected: cancer type, first author, year of publication, country, resources of sample, testing technique of DJ-1, sample size in each groups, age and gender of patients, end point, the source of hazard ratio (HR) (univariate or multivariate analysis), HR and corresponding $95 \%$ confidence interval (CI), tumor-node-metastasis (TNM) classification, tumor differentiation degree, tumor node metastases and the concrete data of DJ-1 expression (number of true positives [TP], false positives [FP], true negatives $[\mathrm{TN}]$ and false negatives $[\mathrm{FN}]$ ).

\section{Quality assessment}

The Newcastle-Ottawa Scale (NOS) was used to evaluate the methodological quality of included studies..$^{25}$ The quality of diagnostic accuracy studies was assessed by the Quality Assessment of Diagnostic Accuracy Studies (QUADAS-2) tool, which comprises four key domains: patient selection, index test, reference standard and flow and timing. ${ }^{26}$

\section{Statistical analysis}

We calculated the combined odds ratio (OR) and corresponding 95\% CI using fixed effects model or random effects model according to the heterogeneity of enrolled studies, which was evaluated by the chi-square value and $I^{2}$ and considered significant at $p<0.1$ and $I^{2}>50 \%$. When the heterogeneity is not significant $\left(I^{2}<50 \%\right.$ and $\left.p>0.1\right)$, we combined the results using fixed effects model (Mantel-Haenszel method). Otherwise, the random effects model (M-H heterogeneity method) was used. ${ }^{27}$ In addition, the summarized HR with 95\% CI was calculated to assess the prognostic effect of DJ-1 expression level on overall survival (OS) in patients 
with malignant tumors. Forest plots were used to clarify the pooled sensitivity, specificity, positive likelihood ratio (PLR), negative likelihood ratio (NLR), diagnostic score, diagnostic OR (DOR) and their 95\% CI. ${ }^{28}$ Sensitivity analysis was performed to assess whether the exclusion of any individual study affects the overall results, and the potential publication bias was checked by Deeks' funnel plot. Statistical analyses were performed using STATA (Version 12.0; STATA Corporation, College Station, TX, USA) and considered statistically significant at the 0.05 level for a two-sided test.

\section{Results}

\section{Search results}

We searched the electronic bibliographic databases and found a total of 383 potentially relevant studies, and 274 studies remained available after duplicate articles were removed. Through a careful screening of the title and abstract among the remaining records, 228 irrelevant studies were excluded and 46 studies were reserved for further assessment. And then we excluded 32 articles due to the studies did not report sufficient data of DJ-1 expression level $(n=19)$ and the sample size was less than $50(\mathrm{n}=13)$ (Table S1). Finally, 14 studies with a total of 1,947 subjects that included 1,410 cancer patients were included in our meta-analysis. The selection progress is shown as a flowchart in Figure 1.

\section{Characteristics of included studies}

Table 1 shows the detailed characteristics of the 14 studies, ${ }^{10-17}$ ${ }^{20-23,29,30}$ which are published from 2010 to 2016, containing a total of 1,410 cancer patients with the sample sizes distributing from 60 to 185 patients. The types of the malignant tumors included GSCC, ${ }^{10} \mathrm{HCC},{ }^{17} \mathrm{NSCLC},{ }^{11}$ cervix squamous cell carcinoma (CSCC), ${ }^{29}$ intrahepatic cholangiocarcinomas (IHCCs), ${ }^{22}$ Glioma, ${ }^{23}$ GC, ${ }^{16}$ triple negative breast cancer (TNBC), ${ }^{12-14}$ medulloblastoma (MB), ${ }^{21}$ nasopharyngeal carcinoma (NPC), ${ }^{30}$ pancreatic neuroendocrine neoplasms $(\mathrm{PNENs})^{20}$ and invasive breast cancer (IBC). ${ }^{15}$ All studies used immunohistochemistry (IHC) method to investigate the expression level of DJ-1 in cancer patient and control. And six studies determined the prognostic value of DJ-1 for OS, two studies for disease-free survival (DFS) and one study for progression-free survival (PFS). The specific information about DJ-1 expression level in the different groups of clinicopathological features is shown in Table 2.

\section{DJ-I expression level between tumor tissues and normal tissues}

Seven studies focused on the expression level of DJ-1 between tumor tissues and normal tissues, ${ }^{10-15,29}$ which included 718 cancer patients and 406 normal controls (Table 2). A fixed effect model was used and the combined OR was $30.72(95 \%$ CI: 20.40-46.26, $p<0.001$ ) (Figure 2A). The pooled results showed statistically significant difference demonstrating that the expression level of DJ-1 was apparently increased in the tumor tissues compared with the normal tissues.

\section{Correlation between DJ-I expression level and clinicopathological parameters}

As shown in Figure 2B and Tables 2 and 3, 11 studies described the association between the expression level of

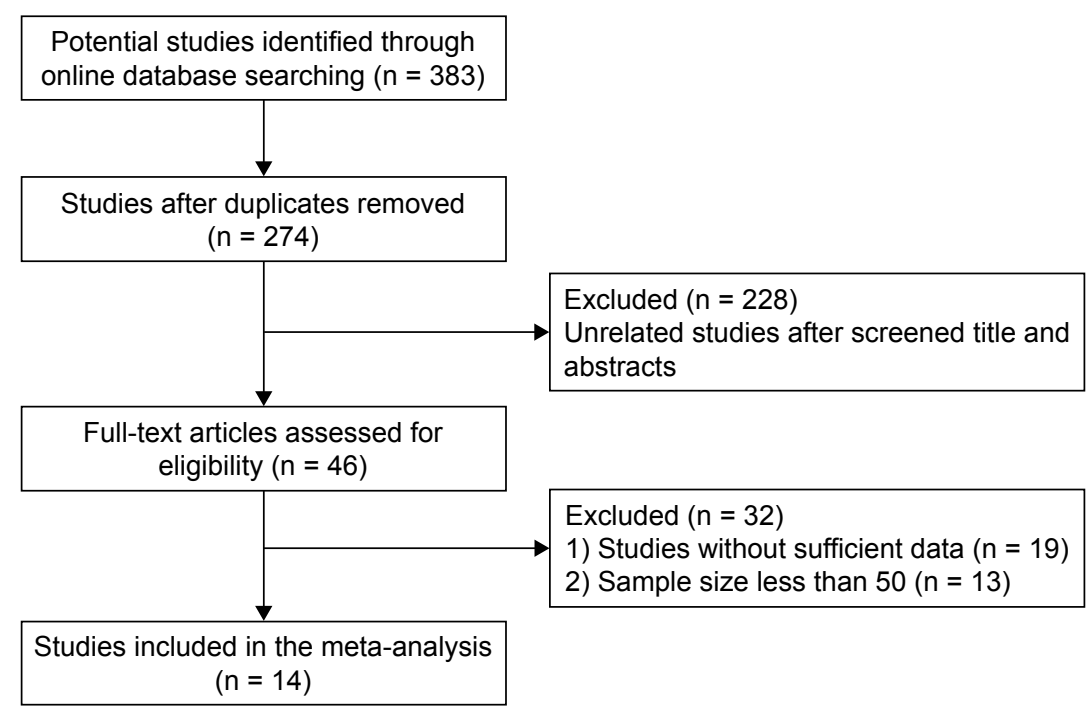

Figure I Flow diagram of the studies identified in the meta-analysis. 


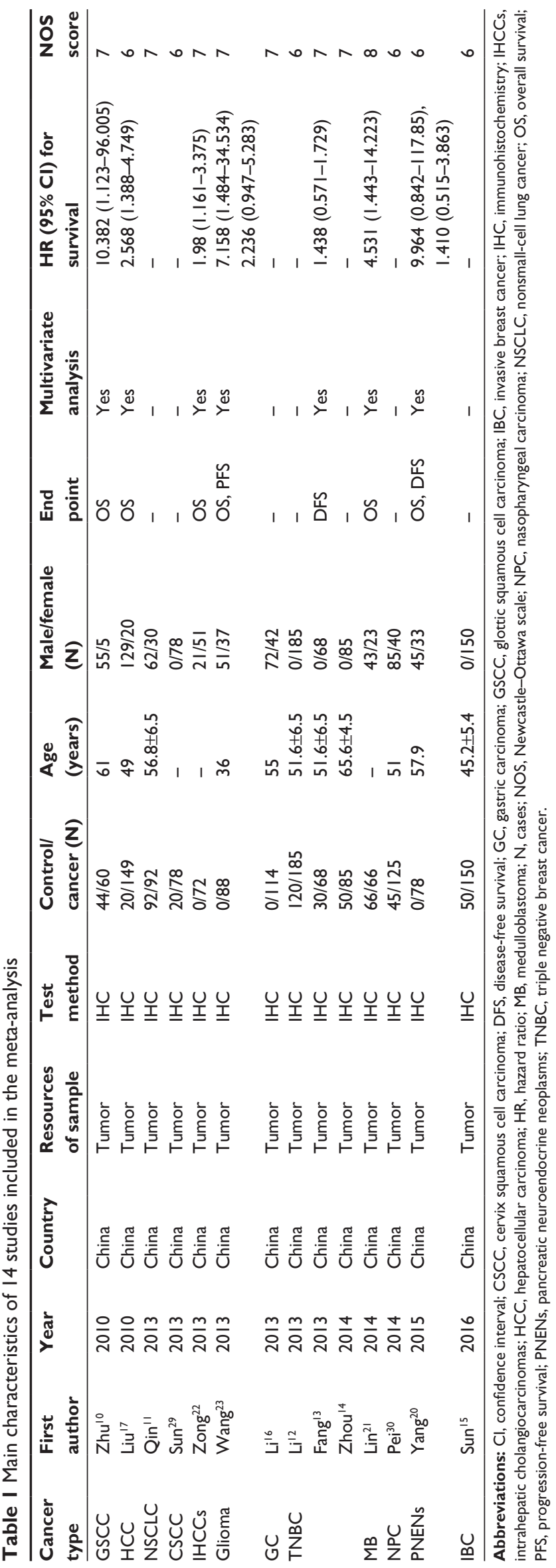

DJ-1 and TNM stage of cancers ${ }^{10-16,20,23,29,30}$ and the pooled OR was $5.52(95 \%$ CI: 3.36-9.05, $p<0.001)$ indicating that increased expression of DJ-1 was obviously correlated with advanced clinical stage of cancers. Similarly, overexpression of DJ-1 was also related to poor differentiation degree $(\mathrm{OR}=2.46,95 \% \mathrm{CI}: 1.08-5.60, p=0.032)($ Figure $2 \mathrm{C})$ and positive lymph node metastasis $(\mathrm{OR}=4.12,95 \% \mathrm{CI}$ : 2.39-7.09, $p<0.001$ ) (Figure 2D).

\section{Correlation between DJ-I expression level and OS}

Six studies clarified the relationship between the expression level of DJ-1 and the OS of cancer patients evaluated by multivariate analysis in the original studies (Table 1). 10,17,20-23 Cancer patients were divided into two groups: DJ-1 high expression group and low expression group. As shown in Figure 3, the combined HR was 2.23 (95\% CI: 1.31-3.14, $p<0.001)$ via a fixed effect model analysis. The pooled results showed that the OS of cancer patients with high DJ-1 expression level is shorter than that with low DJ-1 expression, indicating that DJ-1 can be used as a potential biomarker in predicting prognosis of cancer patients.

\section{Accuracy of DJ-I in the diagnosis of cancers}

Seven studies reported the detailed data about DJ-1 expression level in 718 cancer patients and 406 controls. ${ }^{10-15,29}$ TP, FP, TN and FN were calculated to assess the diagnostic accuracy of DJ-1 (Table 2). As shown in Figure 4, the pooled estimated sensitivity and specificity of DJ-1 for cancer diagnosis were 0.73 (95\% CI: 0.67-0.78) (Figure 4A) and 0.93 (95\% CI: 0.87-0.96) (Figure 4B), respectively. The PLR was 10.11 (95\% CI: 5.59-18.28) (Figure 4C), the NLR was 0.29 (95\% CI: 0.23-0.36) (Figure 4D), the diagnostic score was 3.55 (95\% CI: 2.82-4.29) (Figure 4E) and the DOR was 34.87 (95\% CI: 16.72-72.71) (Figure 4F). The corresponding area under the summary receiver operating characteristic curve (AUC) was 0.88 (Figure 4G). These results suggested a potential diagnostic value of DJ-1 in malignant tumors.

\section{Meta-regression and subgroup analysis}

Significant heterogeneity was observed in the combined results about diagnostic accuracy studies (Figure 4). According to sample size, language and study design, we conducted a meta-regression and subgroup analysis to explore the sources of high heterogeneity. As shown in Figure 5A, the results suggested that the sample size $(p<0.001)$ and study design $(p=0.01)$ may be the sources of heterogeneity for 
Table 2 DJ-I expression in control and cancer patients

\begin{tabular}{|c|c|c|c|c|c|c|c|c|c|c|c|c|c|}
\hline \multirow{3}{*}{$\begin{array}{l}\text { Source } \\
\text { of tumor }\end{array}$} & \multirow{3}{*}{$\begin{array}{l}\text { First } \\
\text { author }\end{array}$} & \multicolumn{8}{|c|}{ Expression of DJ-I (positive/all) (N) } & \multirow{2}{*}{\multicolumn{4}{|c|}{ Diagnostic test }} \\
\hline & & \multirow[t]{2}{*}{ Control } & \multirow[t]{2}{*}{ Cancer } & \multicolumn{2}{|c|}{ Tumor stage } & \multicolumn{2}{|c|}{$\begin{array}{l}\text { Tumor } \\
\text { differentiation }\end{array}$} & \multicolumn{2}{|c|}{$\begin{array}{l}\text { Lymphatic } \\
\text { metastasis }\end{array}$} & & & & \\
\hline & & & & I-II & III-IV & $\begin{array}{l}\text { Well to } \\
\text { moderate }\end{array}$ & Poor & Yes & No & TP & $\mathbf{F P}$ & $\mathbf{T N}$ & $\mathbf{F N}$ \\
\hline GSCC & Zhu' ${ }^{10}$ & $10 / 44$ & $45 / 60$ & $28 / 42$ & $17 / 18$ & - & - & $1 / 2$ & $44 / 58$ & 45 & 10 & 34 & 15 \\
\hline $\mathrm{HCC}$ & $\operatorname{Liu}^{17}$ & - & $87 / 149$ & - & - & $39 / 95$ & $48 / 54$ & - & - & - & - & - & - \\
\hline NSCLC & Qin"l & $7 / 92$ & $67 / 92$ & $40 / 62$ & $27 / 30$ & $36 / 50$ & $31 / 42$ & $37 / 40$ & $30 / 52$ & 67 & 7 & 85 & 25 \\
\hline $\operatorname{cscc}$ & Sun 29 & $0 / 20$ & $67 / 78$ & $42 / 68$ & $8 / 10$ & $38 / 62$ & $12 / 16$ & $48 / 65$ & $2 / 13$ & 67 & 0 & 20 & II \\
\hline IHCCs & Zong ${ }^{22}$ & - & $39 / 72$ & - & - & $16 / 35$ & $23 / 37$ & $17 / 32$ & $22 / 40$ & - & - & - & - \\
\hline Glioma & Wang ${ }^{23}$ & - & $33 / 88$ & $5 / 47$ & $28 / 41$ & - & - & - & - & - & - & - & - \\
\hline $\mathrm{GC}$ & $\mathrm{Li}^{16}$ & - & $76 / 114$ & $19 / 40$ & $57 / 74$ & $46 / 67$ & $30 / 47$ & $55 / 74$ & $21 / 40$ & - & - & - & - \\
\hline \multirow[t]{3}{*}{ TNBC } & $\mathrm{Li}^{12}$ & $3 / 50$ & $63 / 85$ & $26 / 45$ & $37 / 40$ & - & - & $38 / 41$ & $25 / 44$ & 63 & 3 & 47 & 22 \\
\hline & Fang $^{13}$ & $6 / 120$ & $137 / 185$ & $45 / 87$ & $92 / 98$ & $31 / 50$ & $17 / 18$ & $90 / 98$ & $47 / 87$ & 137 & 6 & 114 & 48 \\
\hline & Zhou $^{14}$ & $2 / 30$ & $48 / 68$ & $15 / 32$ & $33 / 36$ & - & - & $33 / 36$ & $15 / 32$ & 48 & 2 & 28 & 20 \\
\hline MB & $\operatorname{Lin}^{21}$ & - & $32 / 66$ & - & - & - & - & - & - & - & - & - & - \\
\hline NPC & $\mathrm{Pei}^{30}$ & - & $94 / 125$ & $29 / 42$ & $65 / 83$ & - & - & $75 / 92$ & $19 / 33$ & - & - & - & - \\
\hline PNENs & Yang $^{20}$ & - & $42 / 78$ & $18 / 42$ & $18 / 24$ & $32 / 64$ & $6 / 8$ & $19 / 27$ & $23 / 51$ & - & - & - & - \\
\hline IBC & Sun ${ }^{15}$ & $4 / 50$ & $92 / 150$ & $47 / 93$ & $45 / 57$ & - & - & $52 / 71$ & $40 / 79$ & 92 & 4 & 46 & 58 \\
\hline
\end{tabular}

Abbreviations: CSCC, cervix squamous cell carcinoma; FN, false negative; FP, false positive; GC, gastric carcinoma; GSCC, glottic squamous cell carcinoma; HCC, hepatocellular carcinoma; HR, hazard ratio; IBC, invasive breast cancer; IHCCs, intrahepatic cholangiocarcinomas; MB, medulloblastoma; N, cases; NPC, nasopharyngeal carcinoma; NSCLC, nonsmall-cell lung cancer; PNENs, pancreatic neuroendocrine neoplasms; TN, true negative; TNBC, triple negative breast cancer; TP, true positive.

pooled sensitivity, and language $(p<0.001)$ and study design $(p<0.001)$ for specificity. In the subgroup with the sample size $\geq 150$, the pooled sensitivity, specificity, PLR, NLR, DOR and AUC were 0.69 (95\% CI: 0.65-0.74), 0.94

\section{A}

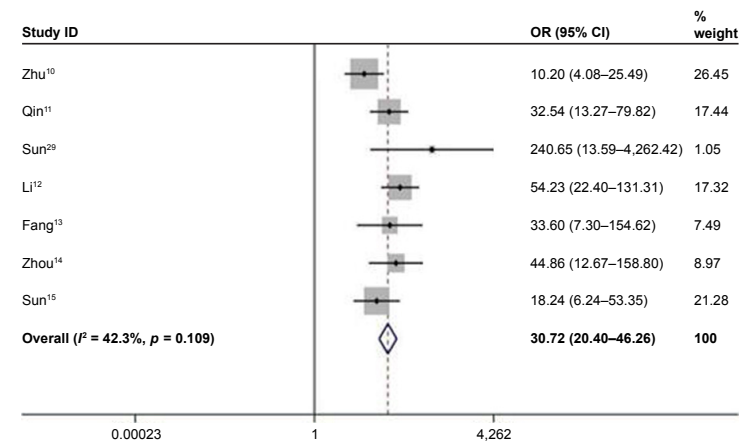

C

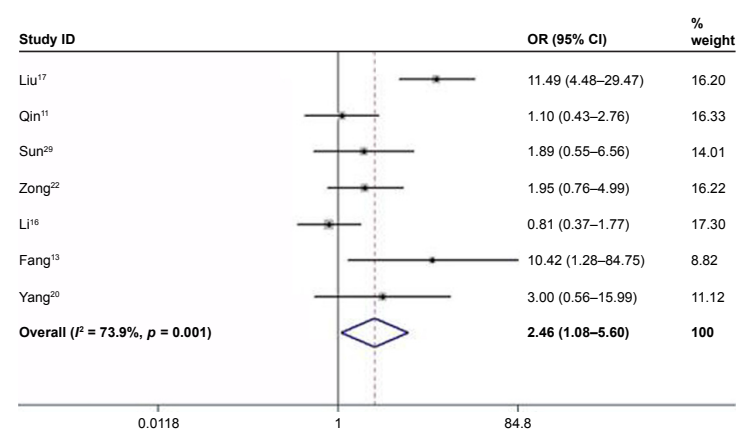

(95\% CI: $0.90-0.96), 10.57$ (95\% CI: $6.65-16.81), 0.33$ (95\% CI: 0.24-0.44), 33.7 (95\% CI: 18.62-61) and 0.95, respectively. And those for subgroup with fewer subjects were 0.77 (95\% CI: 0.71-0.81), 0.90 (95\% CI: 0.83-0.94),
B

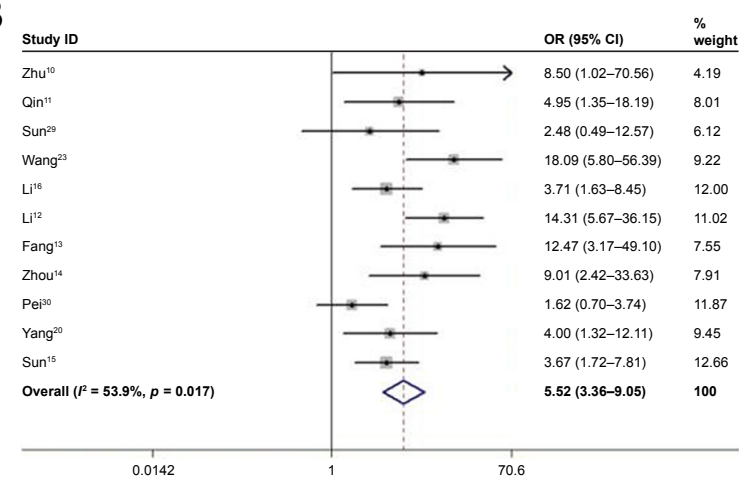

D

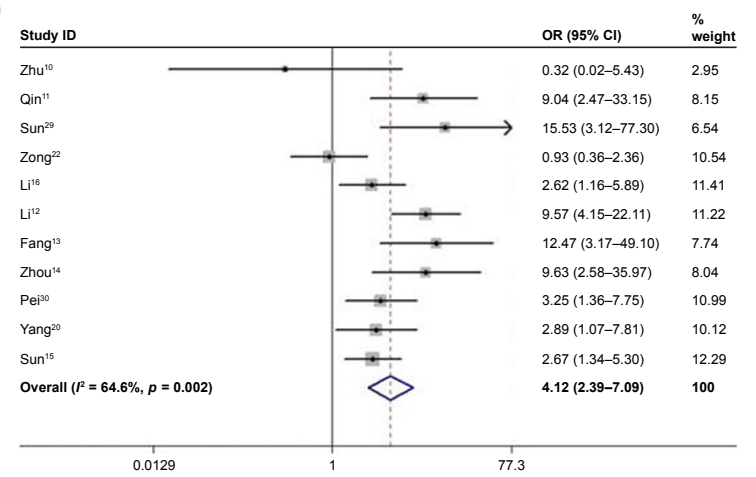

Figure 2 Forest plots of studies evaluating DJ-I expression and clinicopathological features. (A) expression, (B) TNM stages, (C) differentiation and (D) lymph node metastasis. Note: Weights are from random effects analysis.

Abbreviation: TNM, tumor-node-metastasis. 
Table 3 Correlation between increased DJ-I expression and clinicopathological parameters

\begin{tabular}{|c|c|c|c|c|c|c|}
\hline \multirow{2}{*}{$\begin{array}{l}\text { Clinicopathological } \\
\text { parameters }\end{array}$} & \multirow[t]{2}{*}{ References } & \multirow{2}{*}{$\begin{array}{l}\text { Case } \\
\text { number }(\mathbf{N})\end{array}$} & \multirow[t]{2}{*}{ OR $(95 \% \mathrm{Cl})$} & \multicolumn{3}{|c|}{ Heterogeneity } \\
\hline & & & & $I^{2}(\%)$ & $p$-value & Model \\
\hline TNM stage (III-IV vs I-II) & $10-16,20,23,29,30$ & $511 / 600$ & $5.52(3.36-9.05)$ & 53.9 & 0.017 & $\begin{array}{l}\text { Random } \\
\text { effects }\end{array}$ \\
\hline $\begin{array}{l}\text { Histological grade (poor vs well } \\
\text { to moderate) }\end{array}$ & $11,13,16,17,20,22,29$ & $222 / 423$ & $2.46(1.08-5.60)$ & 73.9 & 0.001 & $\begin{array}{l}\text { Random } \\
\text { effects }\end{array}$ \\
\hline Lymph node metastasis (yes vs no) & $10-16,20,22,29,30$ & $578 / 529$ & $4.12(2.39-7.09)$ & 64.6 & 0.002 & $\begin{array}{l}\text { Random } \\
\text { effects }\end{array}$ \\
\hline
\end{tabular}

Abbreviations: $\mathrm{Cl}$, confidence interval; $\mathrm{N}$, cases; OR, odds ratio; TNM, tumor-node-metastasis.

8.46 (95\% CI: 2.75-25.99), 0.26 (95\% CI: 0.19-0.36), 30.19 (95\% CI: 9.8-93.02) and 0.81, respectively (Table 4). Similar results were found in the subgroup analyses according to language and study design (Table 4).

\section{Quality assessment}

We adopted the NOS to assess the quality of the included studies, the results showed that all studies had at least six stars and were considered to be of high quality (Table 1). Seven studies investigated the detailed data about DJ-1 in the diagnosis of malignant tumors, so we used the QUADAS-2 tool to assess the methodological quality of diagnostic accuracy studies and most of studies were assessed to be of low risk and high quality (Figure 6A and B).

\section{Sensitivity analysis and publication bias}

We performed a sensitivity analysis for the clinicopathological parameters due to the relatively obvious heterogeneity.
As shown in Figure 7, the overall results did not change after omitting any one of the involved studies, which suggested that our results were stable and reliable. Deeks' funnel plot (Figure 5B) was conducted to detect the potential publication bias of the diagnostic accuracy studies, and the results showed no publication bias.

\section{Discussion}

DJ-1 is a multifunctional oxidative stress response protein that protects cells from reactive oxygen species and mitochondrial damage. The oxidative modification of cysteine residue (Cys106) is an important posttranslational modification of DJ-1 and allows DJ-1 to act as a sensor of cellular redox homeostasis and determines the cell fate by regulating autophagy or apoptosis in cancers. ${ }^{8,31}$ Accumulating evidences have shown that DJ-1 involves in carcinogenesis of various types of cancer through inhibiting autophagy and apoptosis, which are crucial mechanisms in regulating cell

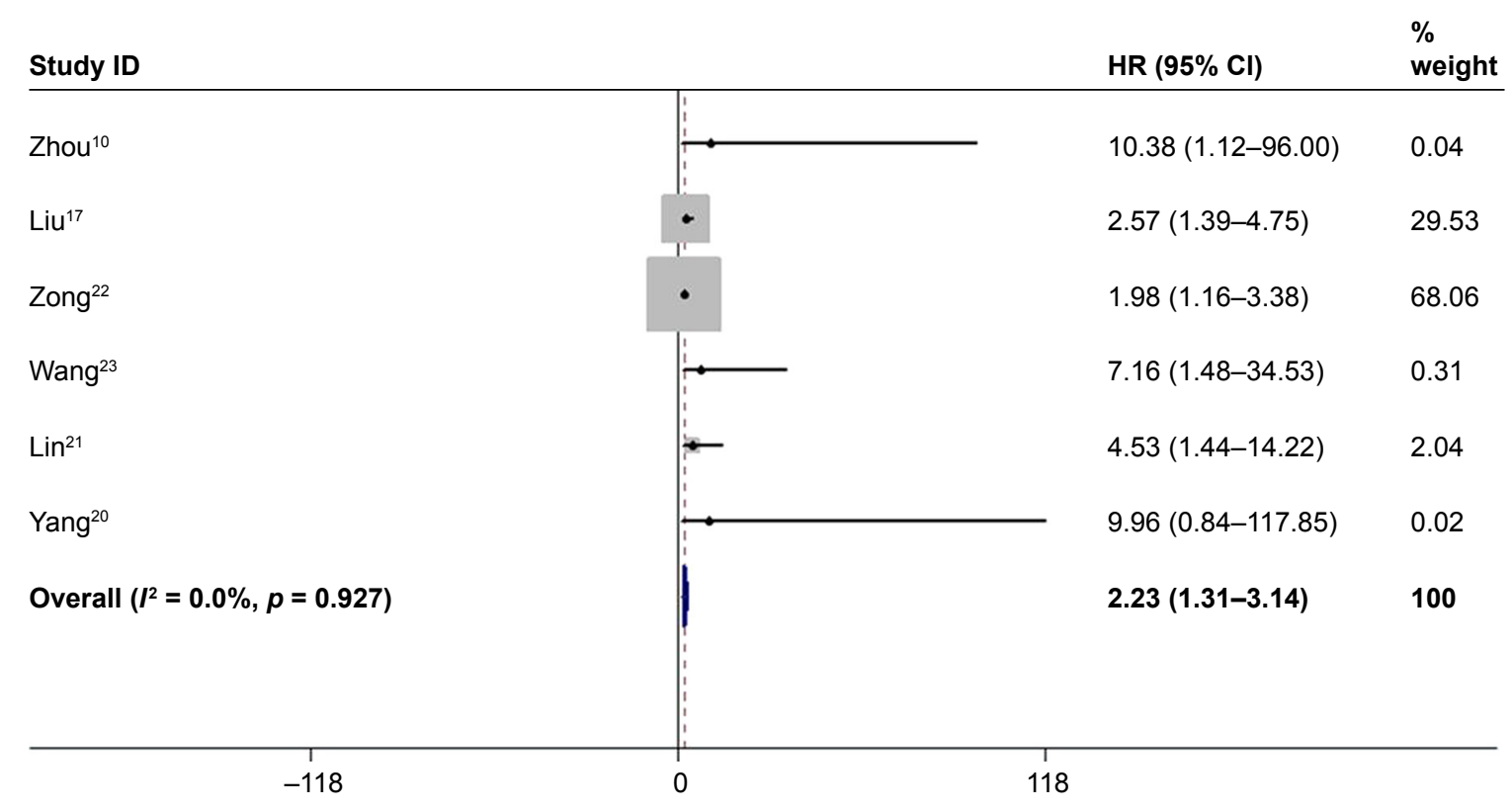

Figure 3 Forest plot of study evaluating DJ-I expression and OS.

Abbreviations: $\mathrm{Cl}$, confidence interval; $\mathrm{HR}$, hazard ratio; OS, overall survival. 
death and homeostasis. Wild-type DJ-1 protein prevents the translocation of death protein Daxx from nucleus to cytoplasm and inhibits the combination and activation of its effector ASK1, thus reducing the cell death. ${ }^{8,32}$ DJ-1 also suppresses the transcriptional activity of p53 and subsequently reduces the expression of Bax and inhibits caspase activity, finally avoiding the occurrence of apoptosis induced by ultraviolet. ${ }^{33}$ Knockdown of DJ-1 gene induces autophagy through activating c-Jun N-terminal protein kinase (JNK) signal pathway and up-regulating the transcription of Beclin 1, which is a mammalian Atg protein and can lead to the generation of autophagosomes and suppress tumorigenesis. ${ }^{34,35}$ Moreover, it has been reported that DJ-1 promotes the proliferation and invasion of laryngeal cancer cells by depressing the expression of PTEN. ${ }^{36,37}$ Using RNA interference technology to silence the expression of DJ-1 could inhibit tumor initiation, invasion and metastasis, suggesting that DJ-1 is a promising therapeutic target in malignant tumors. ${ }^{9,19,38}$ Therefore, DJ-1 is considered as an attractive and important molecular marker in carcinogenesis, proliferation, invasion, metastasis and therapy. We conducted the current meta-analysis to clarify the clinical significances of DJ-1 and to explore whether it could be used as an effective biomarker in clinical practices.

In this meta-analysis, 14 eligible studies with a total of 1,410 cancer patients were eventually included. The pooled results indicated that cancer tissues displayed a higher
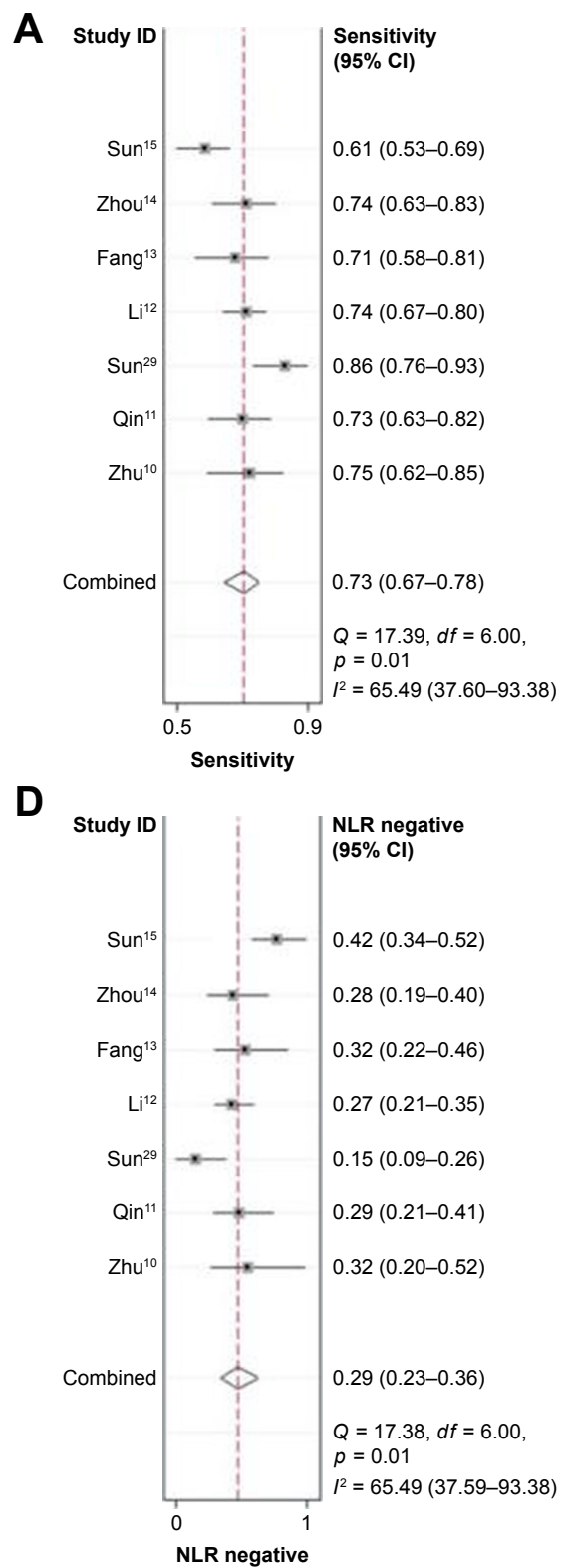

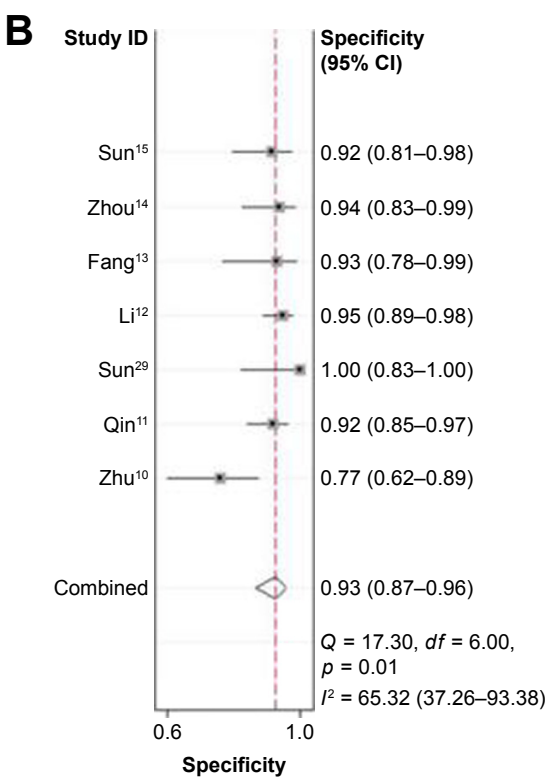

E

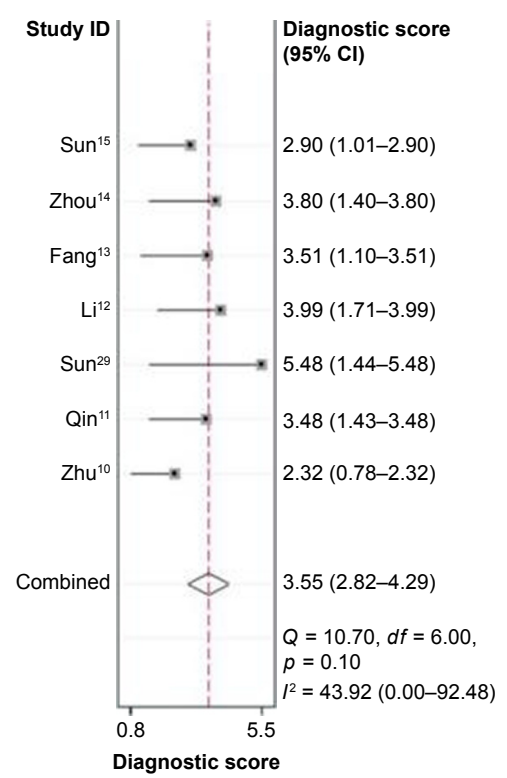

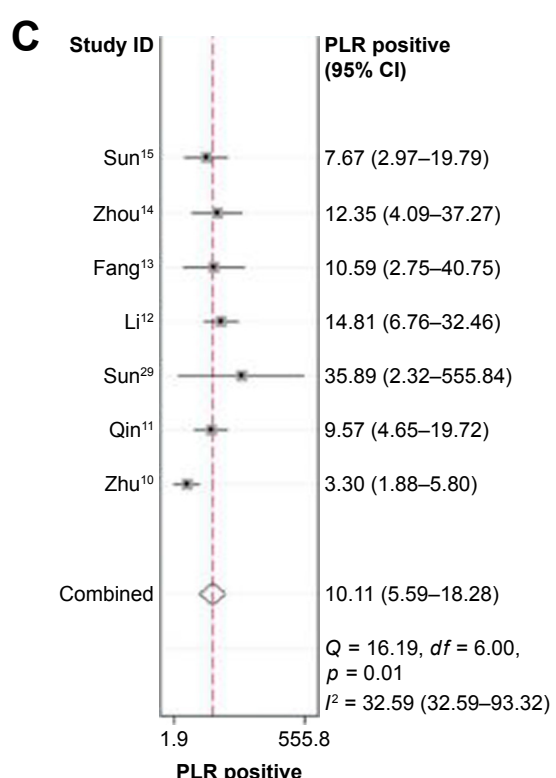

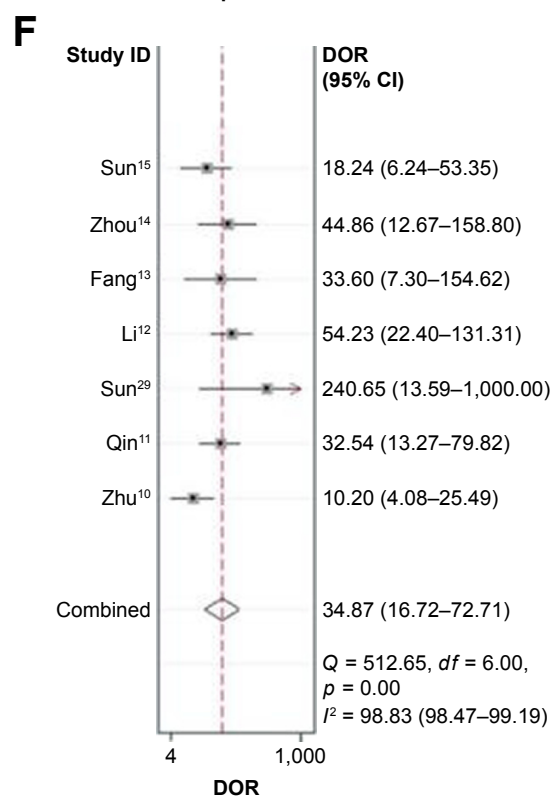

Figure 4 (Continued) 


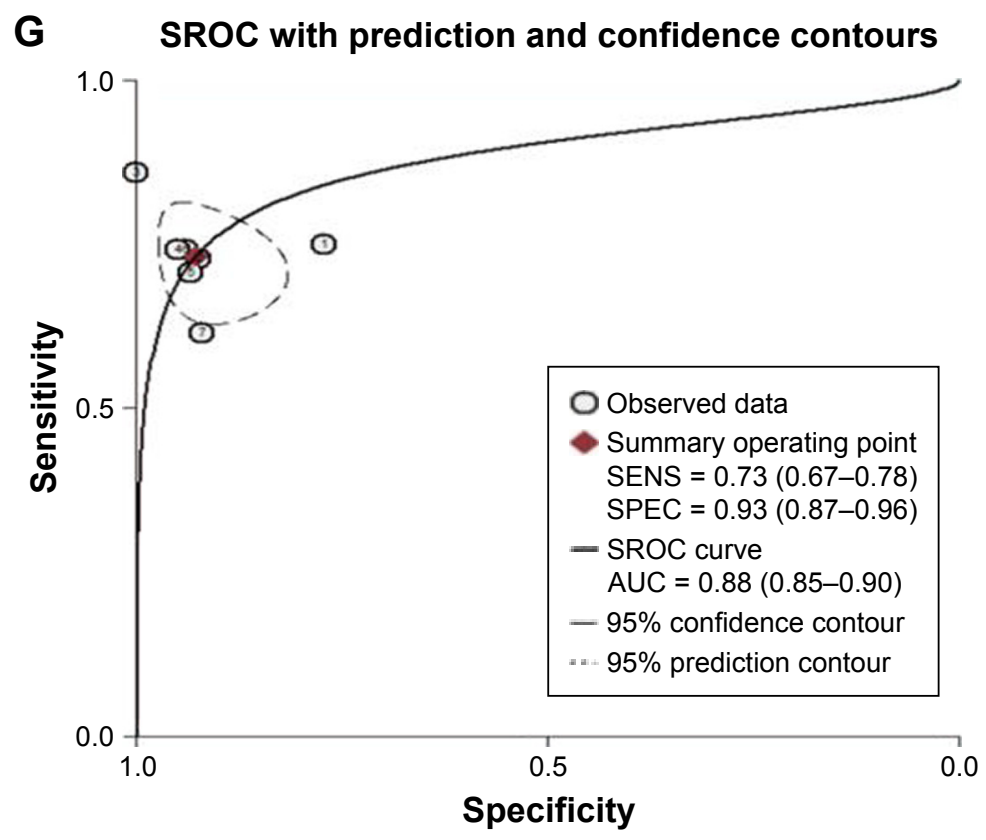

Figure 4 Diagnostic accuracy of DJ-I in cancer tissues. (A) Sensitivity, (B) specificity, (C) PLR, (D) NLR, (E) diagnostic score, (F) DOR and (G) AUC.

Abbreviations: AUC, the corresponding area under the SROC curve; $\mathrm{Cl}$, confidence interval; DOR, diagnostic odds ratio; NLR, negative likelihood ratio; PLR, positive likelihood ratio; SROC, summary receiver operating characteristic curve.

expression level of DJ-1 than normal tissues or adjacent nontumor tissues, and the elevated expression of DJ-1 was obviously correlated with advanced TNM stage cancers, worse histopathological grade cancers and lymphatic invasion-positive cancers, suggesting that DJ-1 was apparently involved in the carcinogenesis and increased DJ-1 was linked to malignant biological behaviors and tumor progression. Furthermore, the pooled HR was 2.23 for OS,
A

\section{Univariable meta-regression and subgroup analyses}

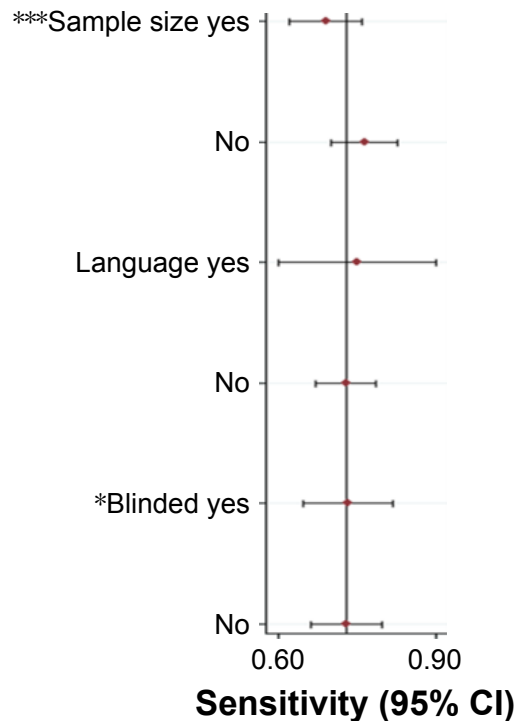

$* p<0.05, * * * p<0.001$

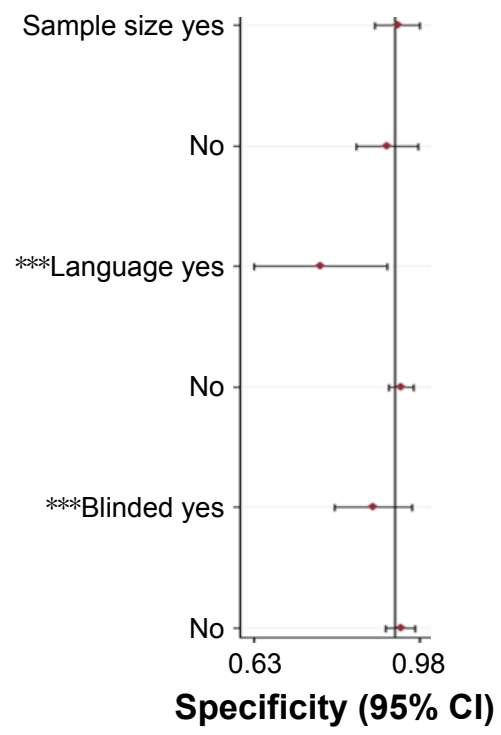

$* * * p<0.001$

\section{B Deeks' funnel plot asymmetry test $p$-value $=0.95$}

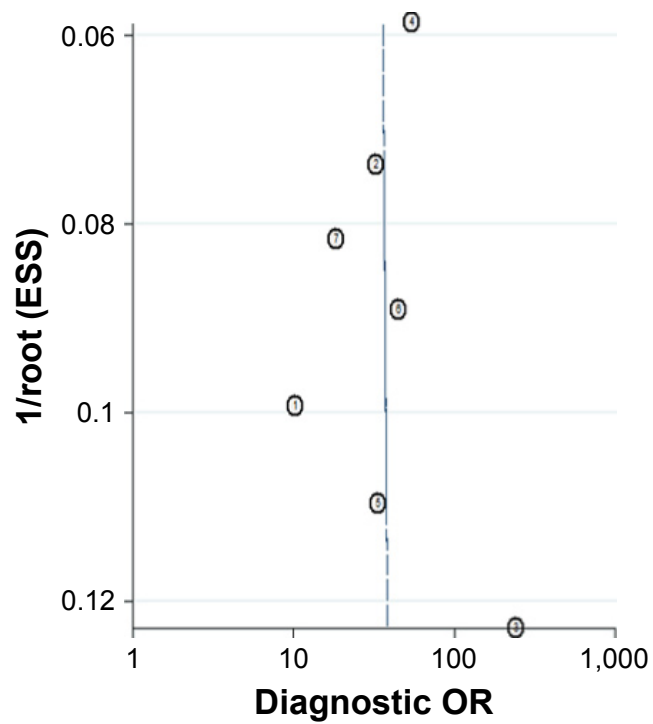

Study ----- Regression line

Figure $\mathbf{5}$ Subgroup analysis and publication bias of diagnostic accuracy studies.

Note: (A) Meta-regression and subgroup analysis and (B) Deeks' funnel plot about the publication bias. Abbreviation: $\mathrm{Cl}$, confidence interval. 


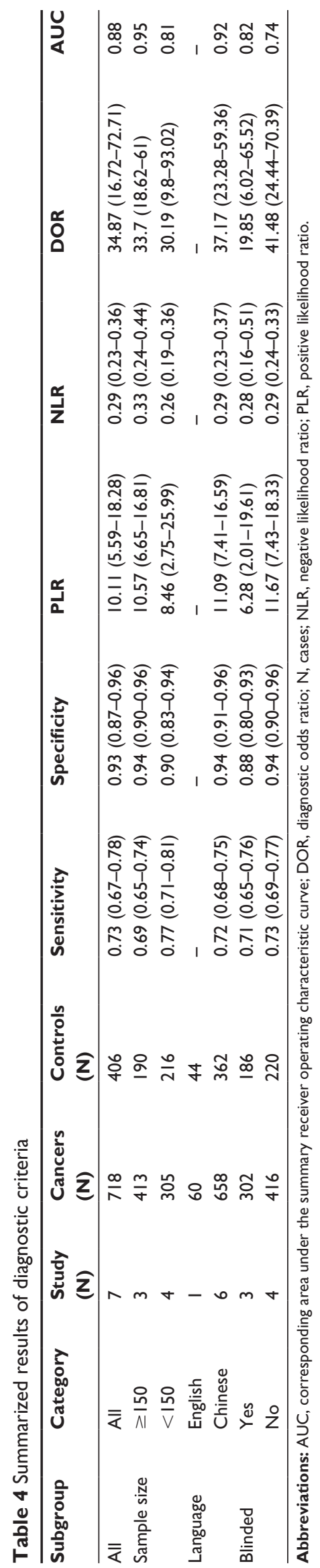

indicating that higher DJ-1 expression level predicted poorer prognosis and it might be a promising biomarker to evaluate the survival rate. Meanwhile, it was also suggested that DJ-1 may be a new potential therapeutic target in malignant tumor and can be used to assess the effect of treatment in cancer patients. However, due to the limited number of studies about DFS/PFS, the correlations between increased DJ-1 and DFS/PFS were not performed, thus further research is warranted to better clarify the prognostic value of DJ-1 in terms of DFS/PFS.

Additionally, DJ-1 had relatively high sensitivity and specificity in the identification of cancers. The PLR was 10.11 , indicating that the chance of DJ-1 being tested positive in cancer patients was 10.11 times compare with the person without cancer. Similarly, the NLR was 0.29 , suggesting that low expression of DJ-1 may be useful to exclude noncancer individuals. DOR is the OR of PLR to NLR, with higher values indicating better discriminatory test performance, ${ }^{39}$ the value of DOR was 34.87 in our meta-analysis, demonstrating that DJ-1 could be used as an effective biomarker to detect malignant tumors. The AUC was used to evaluate the sensitivity and specificity at the same time and it was 0.88 in our study, showing a moderate diagnostic accuracy of DJ-1. Furthermore, we performed Deeks' funnel plot to assess the potential publication bias and did not find any evidence of publication bias. In summarization, DJ-1 could be used as a potential molecular biomarker for cancer diagnosis with high sensitivity and specificity.

There was a significant heterogeneity in our meta-analysis. However, the results of sensitivity analysis for clinicopathological parameters further confirmed the reliability of our results. In addition, we conducted a meta-regression analysis and subgroup analysis to determine the diagnostic accuracy, the results indicated that sample size, language and study design may lead to the heterogeneity in sensitivity and specificity.

To our knowledge, this was the first meta-analysis to assess the expression of DJ-1 and its clinical values in various malignant tumors. However, there were several limitations in the present study. First, the studies defined the positive status of DJ-1 expression using different cutoff values and it may lead to heterogeneity in the pooled results. Second, all the included studies were from China and it required further research in other regions such as Europe, America, Africa and other Asian countries to elucidate the clinical significances of DJ-1. Third, although a comprehensive search of related studies was performed, the number of included study, in particular for each cancer types and for DFS/PFS, was 
A

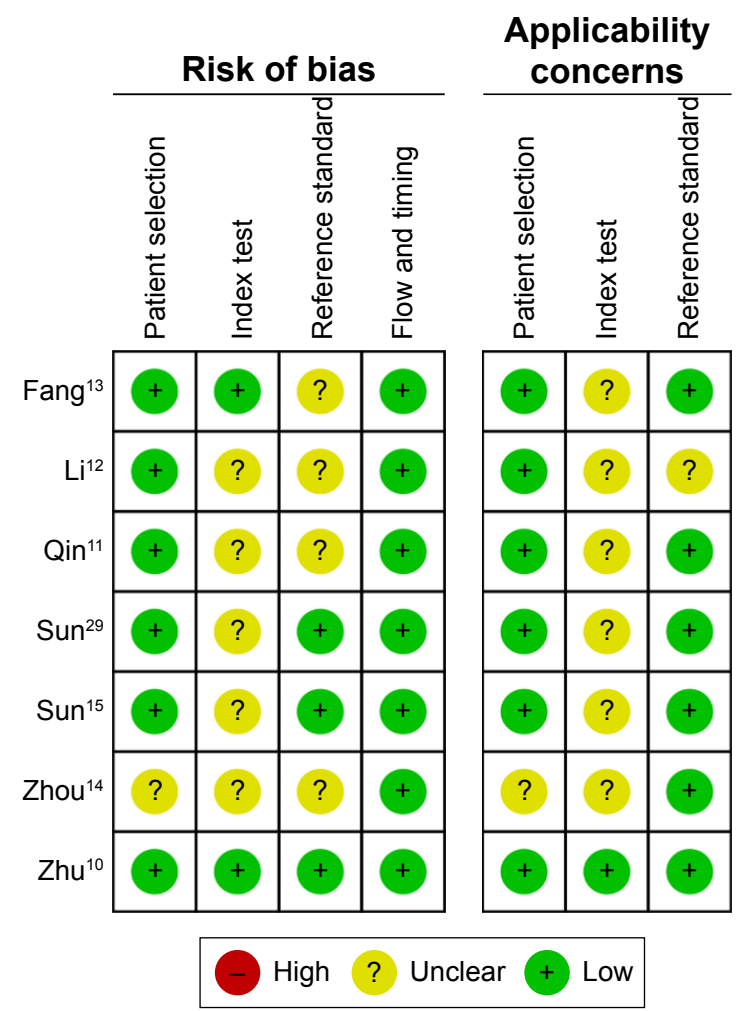

B

Patient selection Index test

Reference standard

Flow and timing
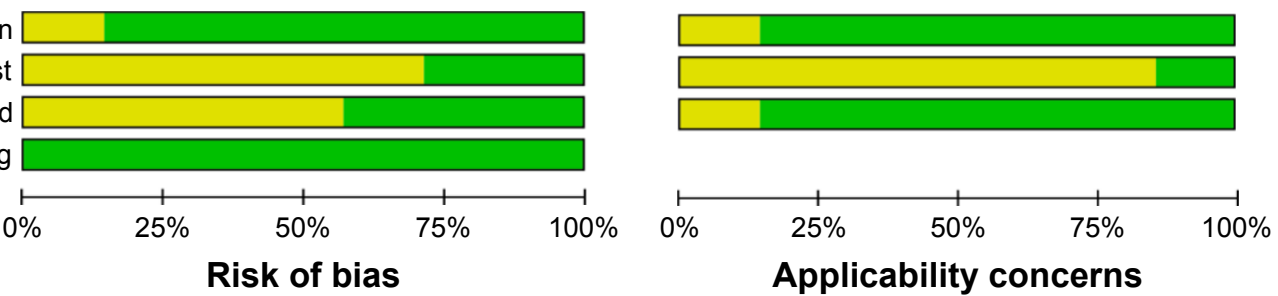

High $\square$ Unclear $\square$ Low

Figure 6 Quality assessments of diagnostic accuracy studies.

Note: (A) Risk of bias and applicability concerns summary and (B) risk of bias and applicability concerns graph.

A

Meta-analysis estimates, given named study is omitted

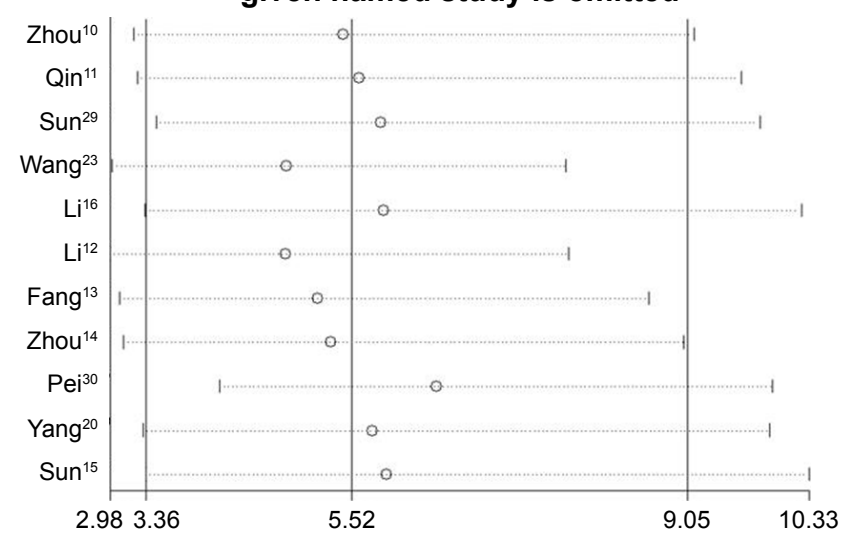

B

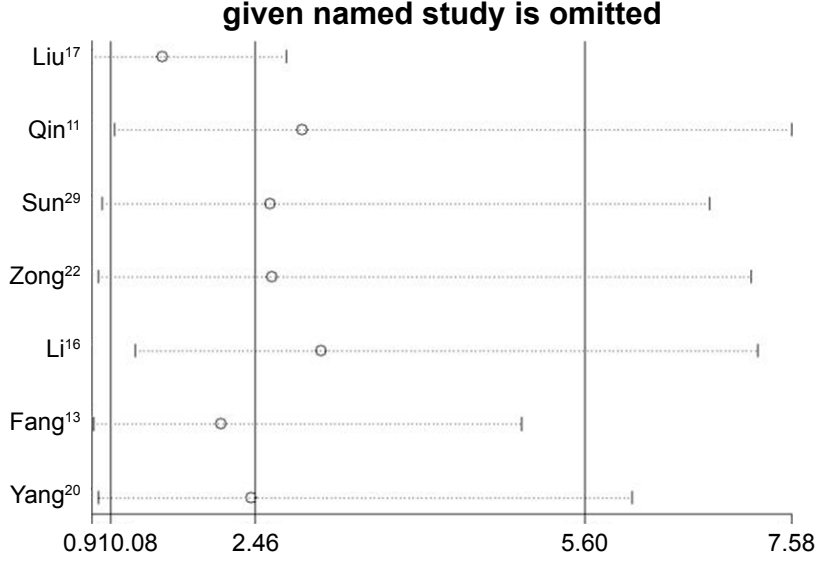

Figure 7 (Continued) 


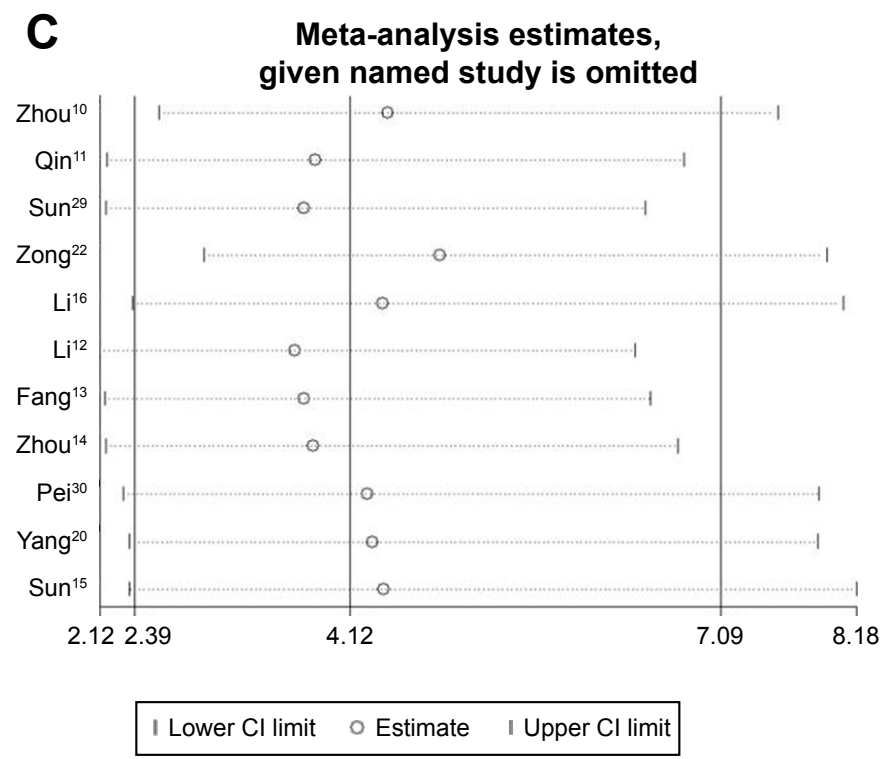

Figure 7 Sensitivity analysis of relationship between increased DJ-I expression and clinicopathological features.

Note: (A) TNM stages, (B) differentiation, and (C) lymph node metastasis.

Abbreviations: $\mathrm{Cl}$, confidence interval; TNM, tumor-node-metastasis.

relatively small and limited. It might weaken the value of our conclusions in some extent and thus large-scale multicenter studies about the expression of DJ-1 are still needed. Fourth, the languages of publications were confined to Chinese and English and it potentially induced a language bias.

\section{Conclusion}

Our meta-analysis revealed that increased expression of DJ-1 was positively correlated with advanced clinicopathological features and poor OS of cancer patients, suggesting that it is an important biomarker in tumor clinical assessments and prognosis prediction. The relatively high sensitivity and specificity of DJ-1 in the detection of tumors indicated that it would be a potential molecular biomarker for cancer diagnosis.

\section{Acknowledgment}

This study was funded by the National Natural Science Foundation of China (grant no. 81670051).

\section{Disclosure}

The authors report no conflicts of interest in this work.

\section{References}

1. Siegel RL, Miller KD, Jemal A. Cancer statistics, 2017. CA Cancer J Clin. 2017;67(1):7-30.

2. Hassanein M, Callison JC, Callaway-Lane C, Aldrich MC, Grogan EL, Massion PP. The state of molecular biomarkers for the early detection of lung cancer. Cancer Prev Res (Phila). 2012;5(8):992-1006.

3. Tanase C, Albulescu R, Codrici E, et al. Circulating biomarker panels for targeted therapy in brain tumors. Future Oncol. 2015;11(3):511-524.
4. Diamandis EP. Cancer biomarkers: can we turn recent failures into success? J Natl Cancer Inst. 2010;102(19):1462-1467.

5. Taira T, Takahashi K, Kitagawa R, Iguchi-Ariga SM, Ariga H. Molecular cloning of human and mouse DJ-1 genes and identification of Sp1-dependent activation of the human DJ-1 promoter. Gene. 2001; 263(1-2):285-292.

6. Le Naour F, Misek DE, Krause MC, et al. Proteomics-based identification of RS/DJ-1 as a novel circulating tumor antigen in breast cancer. Clin Cancer Res. 2001;7(11):3328-3335.

7. Kim RH, Peters M, Jang Y, et al. DJ-1, a novel regulator of the tumor suppressor PTEN. Cancer Cell. 2005;7(3):263-273.

8. Cao J, Ying M, Xie N, et al. The oxidation states of DJ-1 dictate the cell fate in response to oxidative stress triggered by 4-hpr: autophagy or apoptosis? Antioxid Redox Signal. 2014;21(10):1443-1459.

9. Chen Y, Kang M, Lu W, et al. DJ-1, a novel biomarker and a selected target gene for overcoming chemoresistance in pancreatic cancer. $J$ Cancer Res Clin Oncol. 2012;138(9):1463-1474.

10. Zhu XL, Wang ZF, Lei WB, Zhuang HW, Jiang HY, Wen WP. DJ-1: a novel independent prognostic marker for survival in glottic squamous cell carcinoma. Cancer Sci. 2010;101(5):1320-1325.

11. Qin LF, Li YW. Expression of DJ-1 in non-small cell lung cancer and its correlation with clinicopathology. Chin J Gerontol. 2013;2013:4533-4534.

12. Li S, Liu WC. The correlation of DJ-1 and HSP27 expression and clinicopathological features in triple negative breast cancer. $J$ Mod Oncol. 2013;21(06):1231-1234.

13. Fang M, Weng Z, Guan H, Sun Y. Expression of DJ-1, PTEN and AR in triple-negative breast cancer and its correlation with relative clinical parameters and prognosis. Chin J Cancer Prev Treat. 2013;20(10):761-764.

14. Zhou LR, Liu Y. Expression of PTEN and DJ-1 in old triple-negative breast cancer and its correlation with clinicopathology. Pract Prev Med. 2014;21:1242-1244.

15. Sun YH, Guan H, Xu MQ. Expression of DJ-1 and PTEN in invasive breast cancer and its correlation with relative clinical parameters and prognosis. Shenzhen J Integr Tradit Chin West Med. 2016;26(8):9-12.

16. Li Y, Cui J, Zhang CH, et al. High-expression of DJ-1 and loss of PTEN associated with tumor metastasis and correlated with poor prognosis of gastric carcinoma. Int J Med Sci. 2013;10(12):1689-1697.

17. Liu S, Yang Z, Wei H, et al. Increased DJ-1 and its prognostic significance in hepatocellular carcinoma. Hepatogastroenterology. 2010 57(102-103):1247-1256. 
18. Tian M, Cui YZ, Song GH, et al. Proteomic analysis identifies MMP-9, DJ-1 and A1BG as overexpressed proteins in pancreatic juice from pancreatic ductal adenocarcinoma patients. BMC Cancer. 2008;8:241.

19. Hod Y. Differential control of apoptosis by DJ-1 in prostate benign and cancer cells. J Cell Biochem. 2004;92(6):1221-1233.

20. Yang LX, Zhang LN, Zhang TB. The clinical significance of DJ-1 over expression in pancreatic neuroendocrine neoplasms. China Oncol. 2015; 25(2):112-118.

21. Lin JP, Pan BC, Li B, Li Y, Tian XY, Li Z. DJ-1 is activated in medulloblastoma and is associated with cell proliferation and differentiation. World J Surg Oncol. 2014;12:373.

22. Zong M, Jia L, Li L. Expression of novel tumor markers of pancreatic adenocarcinomas in intrahepatic cholangiocarcinomas. Onco Targets Ther. 2013;6:19-23.

23. Wang C, Fang M, Zhang M, et al. The positive correlation between DJ-1 and beta-catenin expression shows prognostic value for patients with glioma. Neuropathology. 2013;33(6):628-636.

24. Liberati A, Altman DG, Tetzlaff J, et al. The PRISMA statement for reporting systematic reviews and meta-analyses of studies that evaluate healthcare interventions: explanation and elaboration. BMJ. 2009;339:b2700.

25. Lo CK, Mertz D, Loeb M. Newcastle-Ottawa scale: comparing reviewers' to authors' assessments. BMC Med Res Methodol. 2014;14:45.

26. Whiting PF, Rutjes AWS, Westwood ME, et al. QUADAS-2: a revised tool for the quality assessment of diagnostic accuracy studies. Ann Intern Med. 2011;155(8):529-536.

27. Higgins JP, Thompson SG, Deeks JJ, Altman DG. Measuring inconsistency in meta-analyses. BMJ. 2003;327(7414):557-560.

28. Deville WL, Buntinx F, Bouter LM, et al. Conducting systematic reviews of diagnostic studies: didactic guidelines. BMC Med Res Methodol. 2002;2:9.

29. Sun YH, Wen W, Song JM, Xu MQ, Yang ZY. Expression of DJ-1 in human cervical lesion tissues and its clinical significance. Chin J Diagn Pathol. 2013;20(3):167-170.
30. Pei XJ, Wu TT, Li B, Tian XY, Li Z, Yang QX. Increased expression of macrophage migration inhibitory factor and DJ-1 contribute to cell invasion and metastasis of nasopharyngeal carcinoma. Int J Med Sci. 2014;11(1):106-115.

31. Wilson MA. The role of cysteine oxidation in DJ-1 function and dysfunction. Antioxid Redox Signal. 2011;15(1):111-122.

32. Junn E, Taniguchi H, Jeong BS, Zhao X, Ichijo H, Mouradian MM. Interaction of DJ-1 with Daxx inhibits apoptosis signal-regulating kinase 1 activity and cell death. Proc Natl Acad Sci U S A. 2005; 102(27):9691-9696.

33. Hinkle DA, Mullett SJ, Gabris BE, Hamilton RL. DJ-1 expression in glioblastomas shows positive correlation with p53 expression and negative correlation with epidermal growth factor receptor amplification. Neuropathology. 2011;31(1):29-37.

34. Ren H, Fu K, Mu C, Li B, Wang D, Wang G. DJ-1, a cancer and Parkinson's disease associated protein, regulates autophagy through JNK pathway in cancer cells. Cancer Lett. 2010;297(1):101-108.

35. Liang XH, Jackson S, Seaman M, et al. Induction of autophagy and inhibition of tumorigenesis by beclin 1. Nature. 1999;402(6762): 672-676.

36. Yao Y, Wei H, Liu L, et al. Upregulated DJ-1 promotes renal tubular EMT by suppressing cytoplasmic PTEN expression and Akt activation. J Huazhong Univ Sci Technolog Med Sci. 2011;31(4):469-475.

37. Zhu XL, Sun W, Lei WB, Zhuang HW, Hou WJ, Wen WP. DJ-1induced phosphatase and tensin homologue downregulation is associated with proliferative and invasive activity of laryngeal cancer cells. Mol Med Rep. 2015;12(2):2003-2008.

38. He X, Zheng Z, Li J, et al. DJ-1 promotes invasion and metastasis of pancreatic cancer cells by activating SRC/ERK/uPA. Carcinogenesis. 2012;33(3):555-562.

39. Bohning D, Holling H, Patilea V. A limitation of the diagnostic-odds ratio in determining an optimal cut-off value for a continuous diagnostic test. Stat Methods Med Res. 2011;20(5):541-550.
OncoTargets and Therapy

\section{Publish your work in this journal}

OncoTargets and Therapy is an international, peer-reviewed, open access journal focusing on the pathological basis of all cancers, potential targets for therapy and treatment protocols employed to improve the management of cancer patients. The journal also focuses on the impact of management programs and new therapeutic agents and protocols on

\section{Dovepress}

patient perspectives such as quality of life, adherence and satisfaction The manuscript management system is completely online and includes a very quick and fair peer-review system, which is all easy to use. Visit http://www.dovepress.com/testimonials.php to read real quotes from published authors. 